\title{
Effective communication regarding risk of fracture for individuals at risk of fragility fracture: a scoping review
}

\author{
Charlotte Beaudart ${ }^{1}\left[\right.$ D $\cdot$ Mickael Hiligsmann ${ }^{1} \cdot$ Nannan $\mathrm{Li}^{1} \cdot$ E. Michael Lewiecki ${ }^{3} \cdot$ Stuart Silverman $^{2}$
}

Received: 5 November 2020 / Accepted: 3 September 2021 / Published online: 24 September 2021

(c) The Author(s) 2021

\begin{abstract}
Summary Two scoping reviews were conducted to review recommendations and guidelines for communication regarding general health risk, and to investigate communication strategies regarding risk of fracture. Healthcare professionals are invited to apply these recommendations to optimize a patient-centered approach to reducing risk of fracture.

Introduction To conduct a scoping review of the medical literature regarding recommendations and tools for effective communication between healthcare professionals and patients regarding general health risk and risk of fracture.

Methods The scoping review was divided into two parts to search for (1) studies presenting recommendations and guidelines for communication regarding general health risk; (2) studies investigating communication regarding risk of fracture for individuals at risk for fractures. Medline was searched in April 2020 to identify relevant studies.

Results The scoping review included 43 studies on communication with regard to general health risk and 25 studies about communication regarding risk of fracture. Recommendations for effective communication with regard to risk are presented. Communication of numeric data on risk should be adapted to the literacy and numeracy levels of the individual patient. Patient understanding of numerical data can be enhanced with appropriate use of visual aids (e.g., pie charts, icon arrays, bar charts, pictograms). The FRAX ${ }^{\circledR}$ tool is the most recommended and most used tool for assessing risk of fracture. Communication sent as individualized letters to patients following DXA scans has been studied, although patient understanding of their risk of fracture is often reported as low using this technique. Use of visual aids may improve patient understanding. Conclusion Healthcare professionals are encouraged to apply recommendations presented in this scoping review in their clinical practice. Patient understanding of risk of fracture should be confirmed by making sure that patients feel free to ask questions and express their concerns. This will contribute to an optimal patient-centered approach. Developing online tools to convert the probability of fracture into patient-friendly visual presentations could facilitate communication between healthcare professionals and patients about risk of fracture.
\end{abstract}

\section{Keywords}

Risk communication, Osteoporosis, Scoping review, Fracture risk.

Charlotte Beaudart

c.beaudart@maastrichtuniversity.nl

1 Department of Health Services Research, CAPHRI Care and Public Health Research Institute, Maastricht University, Maastricht, The Netherlands

2 New Mexico Clinical Research \& Osteoporosis Center, Albuquerque, NM, USA

3 Cedars-Sinai Medical Center, Los Angeles, CA, USA

\section{Introduction}

In shared decision making, the communication process by which patients and clinicians work together to make optimal healthcare decisions is increasingly important. To facilitate this process, decision aids are commonly used to provide information on disease, and on the benefits, and risks of treatment. As part of this, effective communication between healthcare professionals and patients is an important aspect of patient-centered care and shared decision making. Appropriate communication of both healthrelated risks and benefits are essential to help patients make the best-informed health-related decisions that are concordant with their personal values, experiences, and preferences. However, informing patients about their risk of developing a disease, reducing the risk or reaping the 
benefits associated with taking a medication or accepting the risk of side effects associated with a treatment remain challenging. Different intrinsic and extrinsic factors could, at least partially, explain difficulties encountered by patients in understanding information provided by their clinicians. Many patients lack the health literacy needed to understand the words used by clinicians when describing medical information [1]. Clinicians often use medical jargon that is poorly understood by patients. Low numeracy, defined as a low ability to understand numbers and percentages, can also lead to misunderstanding of risk, side effects, and benefits of treatments [2]. Some studies have highlighted observations that perception of risk can be influenced by emotion and by personal experiences [3]. In addition to these intrinsic factors, extrinsic factors may also explain differences in perceptions of risk by healthcare professionals and patients. A hallmark of effective doctor-patient communication on risk is an established relationship of trust [4]. In such a setting, physicians are best equipped to provide understandable and accessible information to patients, with consideration of their numeracy and health literacy levels.

Adequate communication of risk is especially important in the management of patients who are at risk of fragility fractures, such as those living with osteoporosis or osteopenia, where the benefits of treatment and risk of side effects represent key information for making an informed decision. To date, only a minority of patients with fragility fracture are diagnosed or treated appropriately. Patients with fractures may not be aware that they are at risk of subsequent fractures and osteoporosis [5]. Accordingly, from clinical and public health points of view, there is a need for improvement in healthcare professionals' communication to patients regarding risk of fracture, aiming to increase patients' understanding of the risks and consequences of fractures. Fractures may be followed by a cascade of declining mobility, physical activity, muscle strength, quality of life, and balance, contributing to loss of independence and limitation of daily activities that could lead to falls, fall-related injuries including fractures, and in some cases institutionalized medical care [6-8]. Improving patients' knowledge could potentially increase the initiation of and adherence to treatment, and thus lead to fracture prevention.

Recommendations and guidance for successful health risk communication between clinicians and patients have been developed, some of which are focused on communicating risk of fracture within populations at risk. To improve the management of fractures, this study aims to summarize, within a scoping review, recommendations and existing tools for effective communication regarding general health risk, and, more specifically, for communication between healthcare professionals and patients regarding risk of fracture.

\section{Methods}

We performed a scoping review which, in contrast to a systematic review, seeks to present an overview of a large and diverse body of literature pertaining to a broad topic. Within this research method, we seek to provide an overview of communication regarding general health risk. Systematic reviews usually focused on a discrete research question, and produce a summary answer; this was not our objective [9]. Recommendations for the conduct and reporting of scoping reviews were followed throughout the whole procedure of this scoping review (Preferred Reporting Items for Systematic Reviews and Meta-analysis, extension for Scoping Reviews (PRISMA-ScR) checklist) [10]. No protocol was registered, but this is available on request.

\section{Literature search}

The scoping review was organized in two parts. First, we searched for studies presenting methods, rules, recommendations or guidelines for improving communication regarding general health risks. No limit was specified for patient population or disease state. Second, we searched for studies investigating communication on risk of fracture risk (ways to present communication on risk of fracture, recommendations for improving communication on risk of fracture, etc.) in populations at risk of fracture (i.e., with osteoporosis/ osteopenia or with a history of previous fragility fractures).

Medline (via Ovid) was searched in April 2020 to identify relevant studies for both parts of this scoping review. Search strategies are available in Appendix 1. No date restriction was applied and the search was limited to publications in English [11]. All study designs were included with the exception of letters, editorials and case reports.

\section{Study selection and data extraction}

All identified publications were screened for their eligibility by two reviewers (CB and NL), first based on their titles and abstracts and then based on their full texts. Any discrepancies were resolved through discussion between the researchers.

Data were extracted using a standardized extraction form that was pre-tested on a sample of two studies. The following data were extracted from each included publication: authors, year of publication, country (of original paper), study design, type of population, type of risk communication tool, and main results. For better accuracy of data extraction, the data of selected papers were extracted by one reviewer and double-checked by the other. 


\section{Data synthesis}

As the objective of our research was to provide a comprehensive overview of recommendations and existing tools for communicating about risk, a narrative description of results was carried out. We first present studies regarding the first scoping review about recommendations and tools for effective communication of risk in health overall, and then studies reporting on communication regarding risk of fracture.

\section{Results}

\section{Scoping review of communication regarding general health risks}

The search identified a total of 1385 potentially relevant references, of which 120 were included for a full-text review. Of these 120, 77 were excluded because they did not address "communication of risk in general health" between healthcare professionals and patients (i.e., studies on communication of risk of specific diseases, communication of risk during a health crisis, and communication from pharmaceutical industries regarding drug-related risk were all excluded), with 43 studies meeting inclusion criteria. A flowchart of study selection is presented in Fig. 1A. Characteristics and references of included studies are presented in Appendix 2-4.

Included studies were classified into two categories: general recommendations for effective communication of risk and existing tools in communication of risk.

\section{General recommendations for effective communication of risk}

The report of the Medical Device Innovation Consortium (MDIC) entitled "Best practices for communicating benefits, risks and uncertainty for medical device" [12] provides a very complete assessment of benefit and risk communication with evidence-based key factors and available tools for optimal communication. Among the other studies, there were two guidelines that offered general summaries of good practice for communicating risks.

(1) In their guidance to industry "Patient Preference Information - Voluntary Submission, Review in Premarket Approval Applications, Humanitarian Device Exemption Applications, and De Novo Requests, and Inclusion in Decision Summaries and Device Labeling," the Food and Drug Administration concluded that no single format of risk communication between healthcare professionals and patients is universally superior to another format, and therefore developed several recommendations for adequate communication of risk [13] (Table 1).
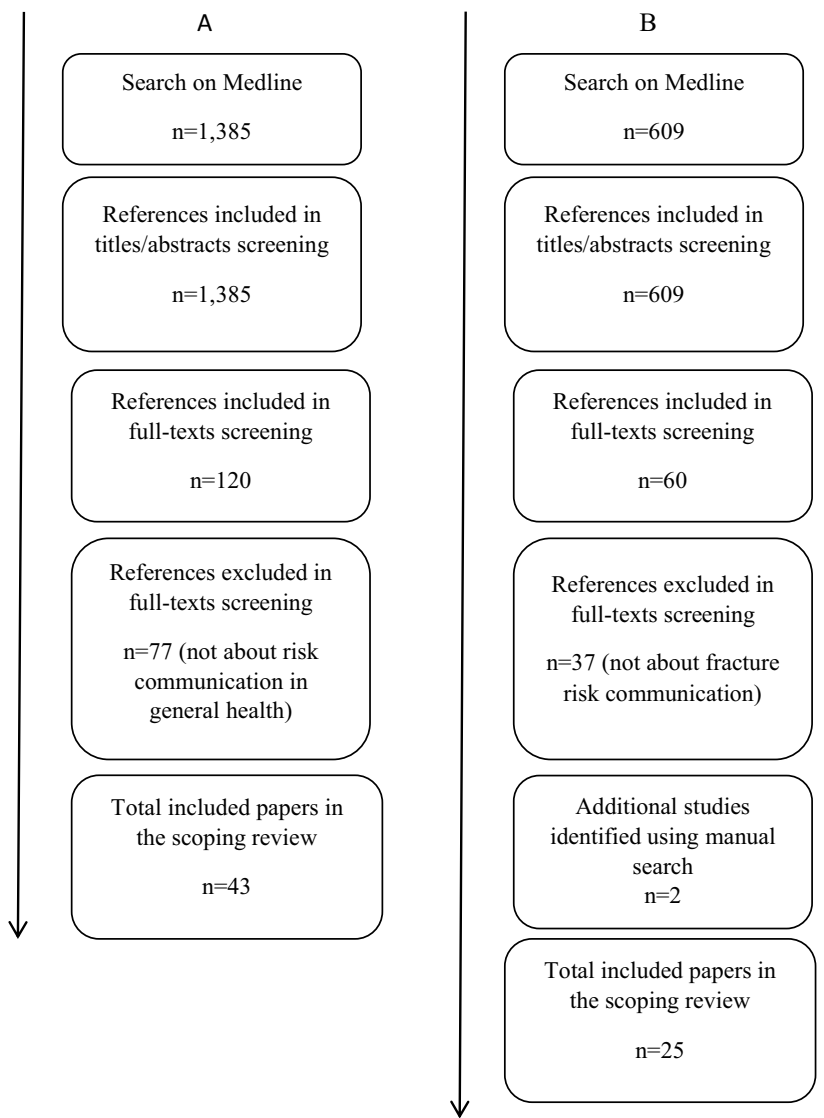

Fig. 1 Flowchart of studies selection for review on communication of risk in general (A) and communication of risk of fracture (B)

(2) Fagerlin et al. [14] proposed 10 steps for better communication of risk with patients, based on an illustrative review of existing evidence-based strategies (Table 2).

Other guidelines, recommendations and rules for effective communication of risk were identified by our scoping review $[3,15]$ and are overall in concordance with the proposals of the two above.

\section{Existing tools in risk communication}

Across these recommendations, some specific tools or aspects of effective communication of risk can be presented in more depth.

\section{Frequency, percentage, numeric data, probabilities}

Communicating numerical concepts to patients can be a challenge. Physicians often presume that their patients understand the subtle nuances of the risks and benefits of a test or treatment. However, it has been shown that numerous patient-related factors, including advanced age [16] or low level of numeracy [17] may impair understanding of evidence-based information. Many patients cannot perform 
Table 1 FDA overall recommendations for communication of risk [13]

- Avoid solely verbal descriptions of uncertainty. Patients may interpret what "low" and "high" risks are differently

- Avoid fractions, decimals, and different denominators when presenting risks of multiple treatments. These are relatively difficult for cognitive processing

- If possible, describe the benefits and risks in absolute scales instead of relative terms, which better inform the actual benefits and risks

- If possible, use multiple formats simultaneously (e.g., verbal frequency, percent, and icon array/pictograph). Relative understanding of these formats varies from patient to patient. Moreover, one format may make the other formats easier to understand

- If possible, describe uncertainty in both positive and negative frames (e.g., $20 \%$ chance of adverse events or $80 \%$ chance of no adverse events) to avoid cognitive bias

- Pretest the communication format. Since patient populations vary, pre-testing the chosen format can improve the comprehension of the format by the study population of interest

Table 2 Fagerlin et al.'s [14] 10 recommendations for effective communication of risk
- Use plain language to make written and verbal materials more understandable

- Present data using absolute risks

- Present information in pictographs if you are going to include graphs

- Present data using frequencies

- Use an incremental risk format to highlight how treatment changes risks from preexisting baseline levels

- Be aware that the order in which risks and benefits are presented can affect risk perceptions

- Consider using summary tables that include all of the risks and benefits for each treatment option

- Recognize that comparative risk information (e.g., what the average person's risk is) is persuasive and not just informative

- Consider presenting only the information that is most critical to the patients' decision making, even at the expense of completeness

- Repeatedly draw patients' attention to the time interval over which a risk occurs basic numeric tasks, and even among highly educated people a study has found that $16 \%$ incorrectly answered a simple question about the magnitude of risk [2].

Communication of risks (e.g., side effects of a drug) can be presented to the patient through a variety of numerical formats. Clinicians could quantify risks using absolute risk, relative risk, attributable risk, odds ratio, hazard ratio, number needed-to-treat, or even survival estimates. For each of these formats, the patients' ability to understand the information presented differs, as does the way the information influences their perceptions of risk [18].

Studies have consistently shown that patients prefer to receive information as absolute risk (e.g., 13 out of 100 will have a fracture) rather than relative risk (e.g., 13\% increased risk of fracture) [14, 15, 19-21]. Communicating the benefits and harms of treatment in the form of relative risk remains even more problematic, even when the baseline risk is explicitly provided and tends to magnify perception of risk [22, 23]. Moreover, several of the included studies showed that the concept of number needed-to-treat (NNT) may not be readily understood by patients, and should be avoided in doctor-patient communication [14, 15, 19, 23]. In the same vein, it has been found that numerical likelihoods presented as 1-in-X (e.g., 1 in 25) were processed quickly and were perceived as conveying larger likelihoods than the $\mathrm{x}$-in-100 (e.g., 4 in 100) and percentage formats (e.g., 4\%) [22, 24]. There is also a preference for using a smaller number for X. Pighin et al. [25] suggested that the 1-in-X format may increase the ability to identify oneself as affected by the possible outcome. There is also general agreement that decimals (e.g., likelihood of 0.03 ) should not be used in communication of risk, as they may lead to misunderstanding [18].Various techniques have been used to reduce requiring inferences and calculations from patients in interpreting risks. For example, when using frequencies, providers should keep the denominator constant to reduce individuals' effort and increase their comprehension. Finally, presenting a higher level of statistical concepts, such as confidence intervals, should also be avoided.

Another concept to consider in numerical risk communication is "denominator neglect." Denominator neglect is a classical bias which can occur when people pay too much attention to the number of times an event happens (numerator) without paying attention to the overall number of opportunities for it to happens (denominator). For example, Yamagishi et al. [26] showed that people rated the likelihood of a cancer killing 1286 out of 10,000 people (i.e., $12.86 \%$ ) as higher than 24.14 out of 100 people (i.e., $24.14 \%$ ). Numerator neglect is more prominent among individuals with low numeracy when risk information is provided numerically. 
Highly numerate people are more likely to pay attention to numbers, understand them better, translate them into meaningful information, and ultimately use them in decisions. Lower numeracy has been shown to be associated with overestimation of risk probabilities, higher susceptibility to factors other than numerical data (e.g., framing, mood states, feedback from others), and greater denominator neglect [27]. Careful attention to the way information is presented should allow everyone, including the less numerate people, to integrate numbers better and use them more effectively in decision making. One solution could be to use different presentations of numerical information with target audiences, i.e., tailor the presentation of numerical information to the audience, to increase the comprehensibility and usability of the information. Other communication tools (e.g., visual aids-see next) can be used in addition to numerical information to communicate better with all populations, whatever their numeracy level. The teach-back method (i.e., method used by healthcare providers to confirm whether a patient understands what is being explained to them by asking them to teach-back the information in their own words) could also be informative in assessing patients' understanding of their health risk $[28,29]$.

\section{Visual aids (pie chart, icon arrays, bar chart, pictograms)}

Presenting probabilistic information using visual formats, such as graphics, pictographs (also called icon arrays) or flow diagrams (i.e., diagram representing pictures of the separate steps of a process in sequential order), in addition to numerical formats, may improve individuals' understanding and influence their decision making [30, 31]. This is particularly true for vulnerable people with low numeracy $[32,33]$. However, communications should not assume that all graphics are more intuitive than numbers or text; interpretation of graphics has been shown to be dependent upon expertise and instruction [34]. Visual displays can also be misused and misrepresent statistical information. When possible, any communication of health risk should be supported by use of different visual aids and ideally be pilot tested before use.

Since there are many types of graphs, it is a challenge to select those that communicate risk most effectively. The type of graph and formatting seems to have an effect on comprehension and behavior. Some studies showed that the formats most easily perceived by patients are bar graphs (vertical bars, horizontal bars) and pictographs $[35,36]$. On the other hand, areal presentations (e.g., pie charts) are less effective [37]. It also appears that the combination of different graphic risk presentation formats seems preferable to a single presentation. In a preference study that compared 6 types of graphic representations, an augmented bar chart combined with a flow diagram seemed to be the preferred combined format for communicating risk [38]. The overall differences in preferences among the 6 formats were statistically significant. However, it is important to stress that using preferred graphs does not necessarily lead to better understanding performance of participants [35].

Depending on the objective of the communication, pictograms can also be useful. While pie graphs and bar charts are superior for accurate judgments about proportions, pictograms are most successful at highlighting the number of people affected, or not affected, by a medical treatment [39]. In various studies, the use of pictograms such as an icon array has been shown to increase the accuracy of both lowand high-numeracy people. The use of pictograms results in a lower risk of misunderstanding in comparison with ratio presentations [40]. For example, in a graph using an icon array (Fig. 2), a display of symbols or figures shows the entire population at risk (the denominator) and highlighted icons show those in whom an event occurred (the numerator). Icon arrays may be an effective method for eliminating denominator neglect [41]. It has been shown that accuracy can be aided by using icon arrays that are arranged as groups in a block rather than being scattered randomly. Moreover, the performance of an icon array also seems dependent on the size of the numerator. For example, when the outcome is less than $100 / 1000$, pictograms are better understood than bar charts. However, for more frequent outcomes (e.g., higher than 100/1000), pictogram arrangements may be complicated and bar charts seems to work better [42].

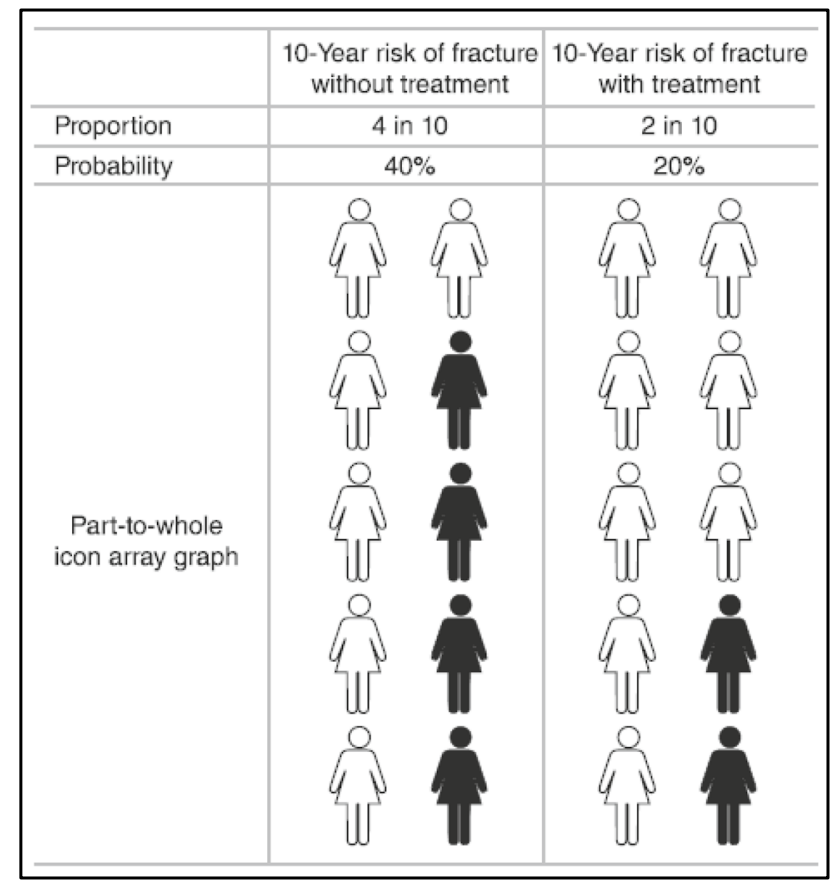

Fig. 2 Expression of risk of fracture using three methods of communication including icon array (Figure issued from Lewiecki et al. [81], used with permission) 
Regarding pictures, a recent focus group study followed by a systematic review [43] explored the impact of different types of pictures (i.e., anatomical pictures, photos, cartoons, pictographs, and drawings) on the perception of health information. However, this study failed to show the superiority of any picture in understanding risk. However, with a moderate quality of evidence, authors recommended that anatomical pictures, cartoons, pictographs, and drawings may be used in addition to text for a better understanding of risks. No recommendation could be given for the use of photos.

Finally, when the information communicated to patients is complex, such as combining numerous risks and benefits, one option for facilitating patients' memory and understanding is to present a visual summary table (i.e., a summary of all of the risks and benefits for each treatment option, presented in a tabular format). Although no study has investigated and proved the impact of this communication tool on general knowledge, some promising results have underlined the greater interest of patients when a visual aid is provided in the format of a summary table [44].

\section{Using words}

Another way of framing information is the use of words in communicating the probabilities of treatment effects. Simplified language (e.g., avoidance of clinical or statistical jargon, use of simple and well-structured sentences) is recommended so people with low literacy can read and understand the information [14].

A range of terms are commonly used in our daily life to describe risk. A systematic review performed in 2014 [45] compared the use of numbers to the use of words in communicating risks. Results indicated that, in comparison with numerical information, verbal descriptors including words such as "common," "uncommon," and "rare" lead to an overestimation of the probability of adverse effects. Interpretation of these terms varies widely from person to person. Authors of this systematic review concluded that providers of consumer health information should quantify treatment effects numerically; this is also recommended by the FDA. Another recent study found considerable variations in the numerical translation of verbal probability by both patients and clinicians, suggesting that verbal probability expression should not be used alone for communication between doctors and patients [46]. Nevertheless, combining verbal and numerical presentation may be useful to better frame the participant's understanding of risk.

Another important aspect of using words is their valence (i.e., negativity or positivity). Different options can be utilized when discussing benefits and risks, such as using positive/gain terms versus negative/loss terms. Researchers found that presenting adverse event information before benefits (compared to benefits before adverse events) lowers the likelihood that subjects will perceive that the benefits outweigh the risks of a proposed new medication [47]. The framing effect is a form of cognitive bias, whereby people respond differently depending on how risk is presented. A recent report revealed that warning patients of the risks of specific side effects increased the incidence of these very side effects (the "nocebo effect"), while positively framing risk information did not [48]. Another example of positive framing is to say that a risk is "uncommon"; hearing this, "9 in 10 people will not be affected," as opposed to saying that the risk is "common"; hearing this, "1 in 10 people will be affected" [48]. It is suggested that positive framing of the possible side effects of a medication in written and verbal communications might "nudge" patients toward accepting treatments where the expected benefits are likely to outweigh the possible risks [48]. This finding was validated in one experimental study involving 375 Polish students who were verbally presented with vignettes describing conversations with patients about the risks of two medical procedures. The risks were presented with a positive ("risk is low" or "risk is not high") or negative ("risk is high" or "risk is not low") valence [49]. Participants receiving a verbal description with a positive valence were more positive about the patient's decision, and evaluated more positively the doctor's and patient's mood than did participants receiving a description with a negative valence [49].

\section{Communication leaflets}

Informational leaflets, brochures, and medication guides about the risks of treatments with prescription drugs are now commonly developed by pharmaceutical companies in response to regulatory agencies. In 2006, a study reviewing 50 leaflets of the most prescribed medicines in England and Wales highlighted substantial variation in the methods used to communicate risk to patients [50]. Moreover, only $8 \%$ of the leaflets provided any form of numerical measure of risk. Communication using patient information leaflets has shown variable results in regard to its efficacy. In a study including 1000 health practitioners, only a small minority of responders correctly stated the meaning of terms that were used to describe the risk presented in a communication leaflet. The idea that event rates are best expressed by numerical values comparing groups with and without an intervention was supported in the findings of a systematic review of studies evaluating risk communication tools [51]. Although most formats increased patients' understanding of risk, they were more likely to do so when structured (whether written, verbal, or video) or interactive (computer, question prompts), particularly when tailored to the needs of the individual receiving the information.

It is also important to keep in mind that presenting less information may be more effective than presenting too much 
information. When designing leaflets or education materials, there is a natural tendency to present a lot of information to include all important data. However, too much information can distract patients and diminish focus on the essential information that is needed for decision making [52]. Catching the essential is therefore an important feature in the communication of risk.

\section{Electronic communication tools}

Risk communication tools other than traditional verbal and written methods have been studied and reported on in the literature. However, the applicability of these techniques in daily clinical practice for the communication of risk between doctors and patients is often uncertain and not fully tested. The increasing prevalence of computers, tablets, and mobile devices creates new opportunities for interactive, web-based formats for communicating probability information. For example, virtual game-like interactions with scenarios have been developed and tested in one study to allow users to experience the probability of a health event [53]. The prototype software developed in the study describes the risk and then provides virtual experience to supplement the description. Pilot results suggest that interactive games are associated with an emotional component that static graphics do not provide, and that this might be useful in communicating risk [53]. Contrarily, another study highlighted that interactive communication, such as flash-based animation pictographs, can distract people from understanding relevant statistical information and may represent worse outcomes than representations using classical static risk graphic formats [54]. While electronic communication tools may have great potential for communicating risk, additional studies are needed to fully evaluate their effectiveness.

\section{Scoping review of strategies for communicating risk of fracture}

Based on our search strategy, a total of 609 references were identified on Medline and 60 were included in the full-text review. Among these 60 studies, 37 were excluded because they were not about "risk communication" and 23 met inclusion criteria. A manual search of the bibliographies of included articles identified two additional references; 25 were finally included in the scoping review. A flowchart for study selection is presented in Fig. 1B. The characteristics and references of included studies are available in Appendix 2.

Included studies were published between 2005 and 2018. Nine of them reported the effects of communicating risk (generally) and patient perception of risk (seven reported results on the importance of communicating risk to patients with osteopenia/osteoporosis and two reported the importance of communicating the risk of subsequent fracture to patients with a history of previous fragility fracture). Among these nine studies, three also reported specific techniques/approaches to communicating risk of fracture, and 16 additional studies exclusively reported specific techniques/approaches to communicating risk of fracture (total papers included in this section $n=19$; population of interest was patients at risk of fracture referred for DXA for osteoporosis screening $(n=9)$, patients with a history of previous fracture $(n=3)$, postmenopausal women $(n=2)$ patients having osteopenia/osteoporosis $(n=1)$, or other (premenopausal women, patients 50 years and more) $(n=4))$.

\section{Summary of research about the effects of communicating risk (generally) and patient perceptions of risk}

A total of nine studies were included in this part; one previous narrative review about the importance of risk communication for patients with osteoporosis [55], one author opinion [56], two experimental studies [57, 58], three qualitative studies [59-61], and two cross-sectional studies [62, 63].

Several tools have been developed and are available for assessing individuals' risk of fractures (e.g., FRAX®, Garvan Fracture Risk calculator, Qfracture). These models produce estimates of the probability of fractures (absolute risk) over a 5- or 10-year period. Two interventional studies, one from Hudson et al. [57] and one from Dunniway et al. [58], evaluated the influence of using FRAX® in patients' healthcare decisions. Both studies found that the use of FRAX® was helpful in improving participants' perception of the risk of fracture, their desire to change bone health habits and acceptance of treatment $[57,58]$. In the studies of Hudson et al. [57] and of Dunniway et al. [58], healthcare professionals shared and discussed both FRAX® and DXA results with participants. The perceived risk of fracture and severity of osteoporosis was addressed by reviewing information about osteoporosis and the consequences of fractures. More specifically, in the study of Dunniway et al. [58], an explanation regarding the purpose of FRAX and the threshold values set for the USA were included in a letter.

In 2010, Lewiecki [55] reported in a review that communication regarding the risk of fracture can be done verbally through clinician-to-patient conversations, sometimes supplemented with written (e.g., brochures, leaflets, graphs), electronic (e.g., computer, video), or hands-on visual aids (e.g., models). Authors reported that the manner in which the information obtained from these tools is communicated to patients is important. Indeed, in two qualitative studies included in the review of Lewiecki [55], authors have shown that the messages patients receive after a fracture are perceived to be inconsistent across healthcare providers $[59,60]$. Sale et al.[60] found that patients perceived few messages about their bone health from a fracture clinic. 
They reported that patients often felt that there was a lack of interest in their bone health and did not grasp the correct message (e.g., patients could remember they were high risk but did not believe it or did not know what they were high risk for). In 2018, Jakobsen et al. [61] also highlighted, through a qualitative study with interviews, the importance of a patient's medical history in understanding the risk of fracture. Authors reported that, for people who had never suffered from fractures, even if they are osteoporotic or osteopenic, the risk of fracture is very difficult to appreciate. In his expert opinion, Ettinger [56] stated that much more than risk numbers are needed and suggested that, ideally, fracture risk tools (e.g., FRAX $®$ ) should be integrated into bone densitometry reporting or incorporated into comprehensive, user-friendly, decision aids.

The lack of communication and poor quality of the written materials for communicating information about the prevention of fractures and/or treatment for osteoporosis has been reported in various studies $[60,62,63]$.

\section{Summary of research about specific techniques/approaches to communicating about risk of fracture}

A total of 19 studies were included in this part: 12 randomized controlled trials [57, 64-74], three pre-post intervention studies $[58,75,76]$, three qualitative studies using interviews [60, 77, 78], and one observational study [79]. A majority of these studies included patients with osteoporosis or who were at high risk of fracture (i.e., patients referred for a DXA scan and osteoporosis screening, postmenopausal women, and patients who had already suffered from a fragility fracture $[n=13])$. Other studies evaluated populations of premenopausal women $(n=1)$ [70], participants 50 years of age and older without any other specificities $(n=3)[64,68$, 69], men receiving androgen deprivation $(n=1)$ [76], and a healthcare physician $(n=1)$ [79].

Letters and educational brochures The most investigated tool for communicating risk of fracture consisted of a letter noting the patient's risk of fracture and an educational brochure mailed quickly after the DXA scan $[58,64,66,68-70$, $75,76]$. This is considered to be a rapid, low-cost, direct-topatient tool for communicating risk of fracture.

In four RCTs [64, 66, 68, 69], researchers sent an educational brochure and a letter with an individual's risk of fracture (the letters presented results of the DXA, the diagnosis of osteoporosis, osteopenia or normal, as well as a graph of the 10-year probability of suffering a major osteoporotic fracture) to patients and they evaluated the impact of this risk fracture communication tool on different outcomes (e.g., calcium and vitamin D intake, better knowledge of osteoporosis, enhancing preventive measures against risk of fractures). Results highlighted modest benefits for this type of communication with regard to osteoporosis knowledge, self-efficacy, and lifestyle behaviors (diet and exercise) in comparison with usual care. They did not show an increase in initiating therapy. In a 10-year follow-up of a 2-year RCT, two different interventions were evaluated [70]: an 8-h Osteoporosis Prevention and Self-Management course, or emailing a brochure with information about osteoporosis. All participants were also informed about their personal risk of fracture (high or normal). There were no differences for most long-term outcomes between the two interventions, although participation in the osteoporosis course led to a more favorable pattern of smoking behavior change in comparison with the intervention using letters. However, being told that the risk of fracture was high was associated with long-term improvement in calcium intake and the use of vitamin D supplements, better smoking behavior, and slower loss of femoral neck BMD in comparison with those who were told that their risk of fracture was normal.

Two additional pre-post interventional pilot studies [75, 76] demonstrated that a personalized letter and an informational brochure improved osteoporosis knowledge, feelings of susceptibility regarding osteoporosis, and better understanding of DXA results. In these pre-post studies, outcomes were measured before and after sending personal letters to all participants.

Pictorial representations of fracture risk The second most commonly investigated tool for communicating risk of fracture consisted of pictorial representations of risk for fractures $[60,67,74,77,78]$.

Three RCTs reported the results of interventions using pictorial representations of risk of fractures [67, 74]. The first one compared presentations that framed the patient's absolute risk of fracture either as the chance of having or not having an event, with the likely benefits of osteoporosis treatment expressed as natural frequencies or numbers needed to treat. This study failed to demonstrate any superiority of a method to impact the participant's perception of the need to get treatment. Participants' intentions about taking medication to prevent fractures were not substantially affected by receiving information about the risk of fracture risk or benefits of treatment. Two other RCTs investigated the effect of an osteoporosis decision aid (including the 10 -year risk of having a bone fracture, measured with the FRAX ${ }^{\circledR}$ calculation and represented using a pictograph) $[72$, 74] and highlighted a positive effect of this decision aid for patients' understanding their 10-year risk of fracture. Patient knowledge and involvement improved with this decision aid, but there was no effect on the initiation of treatment.

Three other qualitative studies $[60,77,78]$ also investigated, through interviews, the impact and understanding of pictorial representation of risk of fracture. In the first study [78], a paper-based patient education tool was developed. 
Patients confirmed that the form and substance of the proposed tool was clear for them and that this tool could be helpful as they considered their decision to take or not take drugs, suggesting that this tool could be an appropriate method for explaining individual risk. A second qualitative study [60] focused on the concept of high risk of future fracture; however, even when using a visual graph highlighting the "high risk" segment, patients often found it to be confusing and interpreted it in different ways. Many patients believed that "high risk" had little relevance to their personal circumstances. Finally, a third study [77] provided preferences of patients $(n=142)$ for four different visual depictions of fracture risk (faces array, arrow, bar and stoplight; Fig. 3), all of them identified through a literature review. Of the four risk depictions investigated, a bar graph was the most preferred. The stoplight color system was regarded as the most "clear," "clean," and "easy to read." The majority of subjects rated the pictogram or face array as the most difficult to understand, as this format does not allow people to quickly ascertain their individual risk category.

\section{Discussion}

This scoping review offers an overview of effective risk communication between healthcare professionals and patients. All studies identified in this review agreed that communication of risk is an essential component in the care of patients. There are very close and strong relationships between the quality of risk communication provided by clinicians and the initiation of treatment and persistence in treatment on the part of the patient.

Although many or most clinicians may feel that shared decision making is already standard in their practice, the evidence suggests that it is still possible to improve communication between clinician and patient or their caregivers such as spouse, children, or friends when the patient has cognitive difficulties.

A growing body of research supports the use of visual presentation of diagnostic and health risk information as an efficient way to communicate risk. Using available and effective educational materials in daily practice to communicate
Fig. 3 Four risk depictions (Figure issued from Edmonds et al. [77], used with permission)

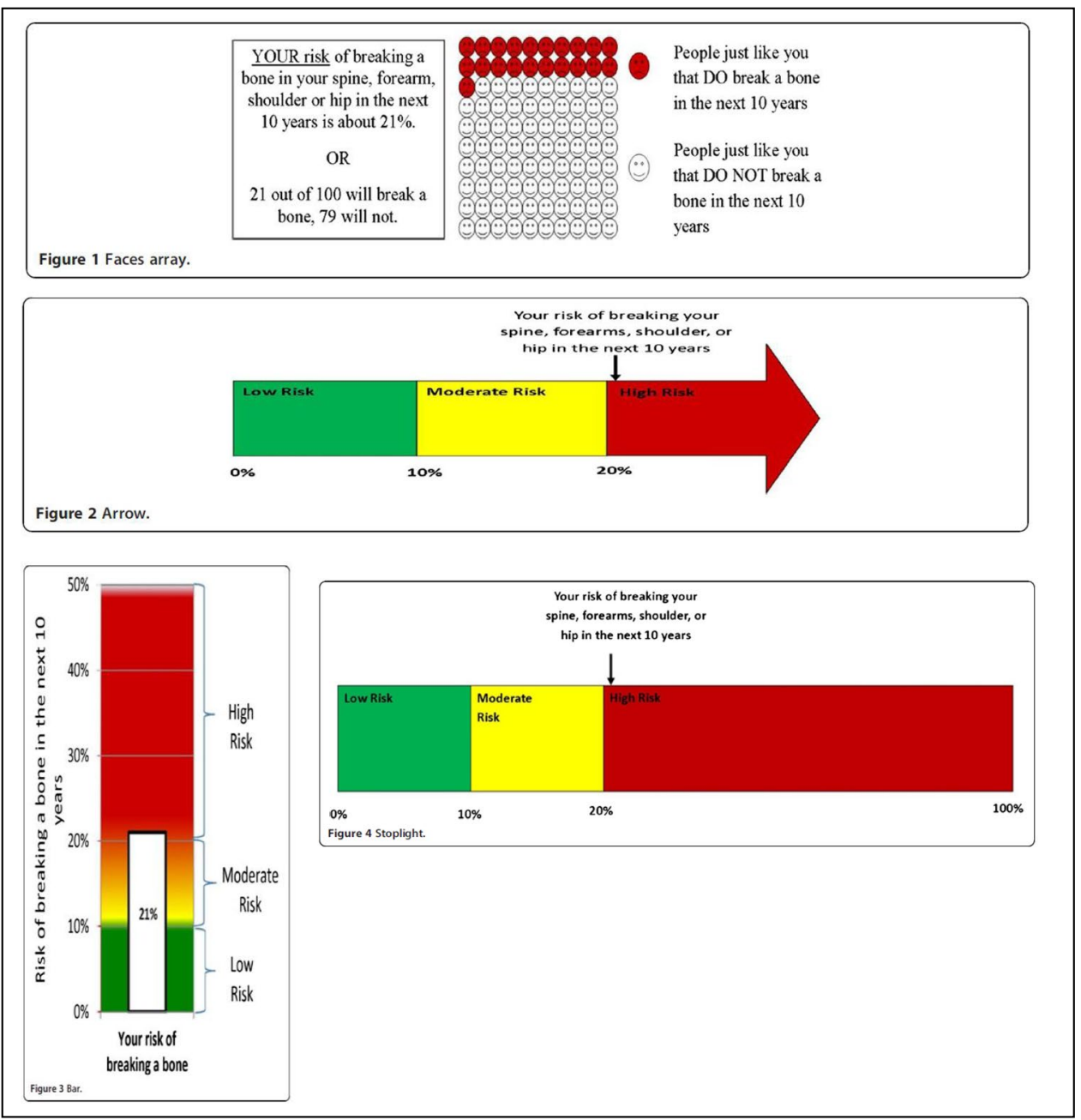


risk in a highly efficient manner could be an important step in enhancing patient education, disease self-management, initiation of treatment, and ultimately persistence in treatment.

Our scoping review has identified various features as contributing to patients' understanding of risk. We identified the ways in which information is presented by clinicians, the ability of the clinician to modify their language according to the needs of the patient, and the relationship between clinicians and patients. We have noted that patients' emotions and self-perceptions of their disease modify the way they understand information on the risk of disease information. Patients need to feel free to ask questions. The way information is understood by patients is limited by factors of health literacy and numeracy as well as biases. All these features need to be considered to successfully communicate risk.

This scoping review also highlights the many ways that risk can be communicated from clinicians to patients. To strengthen communication between clinician and patients, it is recommended that the clinician use plain language, and present numerical data using absolute rather than relative risk. It is also recommended that fractions be avoided; use decimals instead. The sole use of verbal description of risk should also be avoided; use appropriate visual aids such as pie charts, icon arrays, bar charts, or pictograms. It is also recommended that the communication format be tailored to the needs of the individual patient or population.

The development of fracture risk assessment tools such as FRAX®, which calculate absolute risk for fracture over 10 years, offers new opportunities for clinicians to better communicate risk of fracture. However, to date, little has been done to assess the optimal ways to communicate absolute risk of fracture to patients. This scoping review found a general lack of understanding of the most effective ways to communicate absolute risk of fracture and inconsistent understanding of risk of fracture by patients when the risk is presented to them.

The most investigated method for communicating risk of fracture is sending patients an individualized letter, after a DXA test, with information about the risk of fracture and educational material about osteoporosis. However, none of the RCTs comparing this method to usual care were able to demonstrate superiority for change of participants' bone health behavior (e.g., calcium and vitamin D intake, enhancing preventive measures against risk of fractures) or for understanding the risk of fracture. This may be because a letter format is simply not effective or because the written content of the letter is poorly expressed and/or not well understood by the patient. Moreover, other types of educational interventions, such as brochures or websites, are often inadequate and do not always provide evidence-based information and or effectively communicate risk. Quality of understanding using this written approach may therefore be limited. Conversations with patients can be enhanced with decision aids, assuming they are accurate and unbiased in communicating the desired information. Numeric data (e.g., frequencies, percentage, probabilities data) in risk communication is often challenging and should be adapted to the literacy levels of patients. Because health statistics are commonly misunderstood by patients, graphs, or other pictorial representations may be useful in presenting complex numerical information. Based on our scoping review, graphs and stoplight color systems seem to be the most preferred and understandable visual methods for communicating information about risk of fracture. Pictograms with faces, colored in red in case of risk, are rated as the most difficult to understand method. This format does not seem to allow people to quickly ascertain their individual risk category [77]. Another innovative educational material, 3-D printed bone models, was tested in one study [71]. Individuals receiving an interview augmented by the presentation of these 3-D bone models, following their DXA scans, were more emotionally affected by osteoporosis and reported a greater understanding of osteoporosis. It is important to note that these new techniques of communication, even if they improve the quality of communication between healthcare professionals and patients, have not yet demonstrated beneficial effects on initiation of treatment.

In this scoping review, we did not identify any original studies that looked at whether there could be any differences between successful communication with patients having osteoporosis/osteopenia (based on their $T$-score, without a previous fracture) and patients with a history of previous fragility fracture (regardless of $T$-score/diagnosis of osteoporosis). As both populations could potentially differ in their preferences and needs, to improve appropriate use of tools for communicating risk of fracture, studies aiming to determine whether similar or different messages are required for these two different populations would therefore be needed. Well-designed scoping reviews provide comprehensive assessments of topics of interest and may be especially useful in providing an overview of available research evidence when more evidence is needed to answer a specific clinical question.

Although we carefully followed the PRISMA-ScR statement, our methodology nevertheless has some limitations. First, given the objective of this study, we investigated only one bibliographic database. Nevertheless, we performed a deeper manual search to identify the maximum available evidence. Second, because of logistical organization, data extraction was performed by only one investigator. Therefore, we could be prone to bias in collection of data. Nevertheless, a second reviewer carefully checked all extracted data to minimize possible bias. Finally, we did not measure the quality of all individual studies involved in this scoping review. Because of the large heterogeneity of included study 
designs, quality appraisal of individual studies is difficult to standardize and is not mandatory.

Nevertheless, to our knowledge, this is the first scoping review summarizing evidence on effective tools for communicating risk of fracture. This study could therefore be helpful in improving the way healthcare professionals communicate with patients. The final goal may be enhanced by shared decision making, "an approach where clinicians and patients share the best available evidence when faced with the tasks of making decisions, and where patients are supported to consider options, to achieve informed preferences" [80]. To encourage and support informed decision making, healthcare professionals are encouraged to apply the various recommendations presented in this scoping review. In the field of communicating risk of fractures, further studies are needed to offer a better comprehensive approach to optimal communication. Moreover, cultural differences among patients must be recognized and appreciated so that communication of risk can be customized as appropriate. We look forward to the development of improved user-friendly tools to facilitate communication of the risk of fracture. One of our suggestions would be that risk of fracture derived from FRAX ${ }^{\circledR}$ (or other risk algorithms) could be instantly converted to a pictorial representation (e.g., a spotlight bar chart) of the risk, which can be shown and explained to the patient directly following their DXA scan.

\section{Conclusion}

Healthcare professionals are encouraged to apply the recommendations presented in this scoping review to develop a patient-centered approach to communicate risk. A patientcentered approach to communication about risk of fracture includes individualization of the communication format based on the individual patient needs, confirmation by the patient that they understand their risk of fracture and that they feel free to ask questions and express their concerns. A patient-centered approach also includes the development of visual aids based on use of risk of fracture algorithms to effectively express risk of fracture.

Supplementary Information The online version contains supplementary material available at https://doi.org/10.1007/s00198-021-06151-7.

Funding This study is part of the RICO (Risk Communication in Osteoporosis) project that has received funding from Amgen US.

Data availability Under request.

\section{Declarations}

Conflict of interest None.
Open Access This article is licensed under a Creative Commons Attribution-NonCommercial 4.0 International License, which permits any non-commercial use, sharing, adaptation, distribution and reproduction in any medium or format, as long as you give appropriate credit to the original author(s) and the source, provide a link to the Creative Commons licence, and indicate if changes were made. The images or other third party material in this article are included in the article's Creative Commons licence, unless indicated otherwise in a credit line to the material. If material is not included in the article's Creative Commons licence and your intended use is not permitted by statutory regulation or exceeds the permitted use, you will need to obtain permission directly from the copyright holder. To view a copy of this licence, visit $\mathrm{http} / / /$ creativecommons.org/licenses/by-nc/4.0/.

\section{References}

1. Cokely ET, Feltz A, Ghazal S, et al (2018) Skilled decision theory: from intelligence to numeracy and expertise. In: Cambridge Handb. Expert. Expert Perform. Cambridge University Press, pp 476-505

2. Lipkus IM, Samsa G, Rimer BK (2001) General performance on a numeracy scale among highly educated samples. Med Decis Mak 21:37-44. https://doi.org/10.1177/0272989X0102100105

3. Fischhoff B, Brewer N, and Downs J E Communicating risks and benefits: an evidence-based user's guide [Internet]. Silver Spring, MD U.S. Dep. Heal. Hum. Serv. Food Drug Adm. 2011 Aug [cited 2013 Feb 27]. Available fr

4. Chandra S, Mohammadnezhad M, Ward P (2018) Trust and communication in a doctor- patient relationship: a literature review. J Healthc Commun. https://doi.org/10.4172/2472-1654.100146

5. Hooven FH, Adachi JD, Adami S et al (2009) The Global Longitudinal Study of Osteoporosis in Women (GLOW): rationale and study design. Osteoporos Int 20:1107-1116. https://doi.org/10. 1007/s00198-009-0958-2

6. Barrett-Connor E, Sajjan SG, Siris ES et al (2008) Wrist fracture as a predictor of future fractures in younger versus older postmenopausal women: results from the National Osteoporosis Risk Assessment (NORA). Osteoporos Int 19:607-613. https://doi.org/ 10.1007/s00198-007-0508-8

7. O'Reilly C, Keogan F, Breen R et al (2013) Falls risk factors and healthcare use in patients with a low-trauma wrist fracture attending a physiotherapy clinic. Int J Ther Rehabil 20:480-486. https:// doi.org/10.12968/ijtr.2013.20.10.480

8. Hakestad KA, Nordsletten L, Torstveit MK, Risberg MA (2014) Postmenopausal women with osteopenia and a healed wrist fracture have reduced physical function and quality of life compared to a matched, healthy control group with no fracture. BMC Womens Health 14:92. https://doi.org/10.1186/1472-6874-14-92

9. Pham MT, Rajić A, Greig JD et al (2014) A scoping review of scoping reviews: advancing the approach and enhancing the consistency. Res Synth Methods 5:371-385. https://doi.org/10.1002/ jrsm. 1123

10. Tricco AC, Lillie E, Zarin W et al (2018) PRISMA extension for scoping reviews (PRISMA-ScR): checklist and explanation. Ann Intern Med 169:467-473. https://doi.org/10.7326/M18-0850

11. Morrison A, Polisena J, Husereau D et al (2012) The effect of english-language restriction on systematic review-based metaanalyses: a systematic review of empirical studies. Int J Technol Assess Health Care 28:138-144. https://doi.org/10.1017/S0266 462312000086

12. Science of Patient Input Communication Working Group Consortium of the Medical Device Innovation. Best Practices for Communicating Benefit, Risk, and Uncertainty for Medical 
Devices. https://mdic.org/resource/best-practices-for-communicat ing-benefit-risk-and-uncertainty-for-medical-devices/. Accessed Sept 2020

13. FDA. Patient preference information - voluntary submission $\mathrm{R}$ in PAA, humanitarian device exemption applications, and de novo requests and I in DS and, device labeling. https://www.fda.gov/ media/92593/download Published October 2016. Accessed September 2020. No Title

14. Fagerlin A, Zikmund-Fisher BJ, Ubel PA (2011) Helping patients decide: ten steps to better risk communication. J Natl Cancer Inst 103:1436-1443. https://doi.org/10.1093/jnci/djr318

15. Schrager $\mathrm{S}$ (2018) Five ways to communicate risks so that patients understand. Fam Pract Manag 25:28-31

16. Park DC, Lautenschlager G, Hedden T et al (2002) Models of visuospatial and verbal memory across the adult life span. Psychol Aging 17(2):299-320. https://doi.org/10.1037/0882-7974.17.2. 299

17. Schwartz LM, Woloshin S, Black WC, Welch HG (1997) The role of numeracy in understanding the benefit of screening mammography. Ann Intern Med 127:966-972. https://doi.org/10.7326/ 0003-4819-127-11-199712010-00003

18. Sheridan SL, Pignone MP, Lewis CL (2003) A randomized comparison of patients' understanding of number needed to treat and other common risk reduction formats. J Gen Intern Med 18:884 892. https://doi.org/10.1046/j.1525-1497.2003.21102.x

19. Zipkin DA, Umscheid CA, Keating NL et al (2014) Evidencebased risk communication: a systematic review. Ann Intern Med 161:270-280. https://doi.org/10.7326/M14-0295

20. Akl EA, Oxman AD, Herrin J et al (2011) Using alternative statistical formats for presenting risks and risk reductions. Cochrane Database Syst Rev. https://doi.org/10.1002/14651858.cd006776. pub2

21. Covey J (2007) A meta-analysis of the effects of presenting treatment benefits in different formats. Med Decis Mak 27:638-654. https://doi.org/10.1177/0272989X07306783

22. Bodemer N, Meder B, Gigerenzer G (2014) Communicating relative risk changes with baseline risk: presentation format and numeracy matter. Med Decis Mak 34:615-626. https://doi.org/10. 1177/0272989X14526305

23. Trevena LJ, Zikmund-Fisher BJ, Edwards A et al (2013) Presenting quantitative information about decision outcomes: a risk communication primer for patient decision aid developers. BMC Med Inform Decis Mak 13:S7. https://doi.org/10.1186/ 1472-6947-13-S2-S7

24. Cheung YB, Wee HL, Thumboo J et al (2010) Risk communication in clinical trials: a cognitive experiment and a survey. BMC Med Inform Decis Mak. https://doi.org/10.1186/1472-6947-10-55

25. Pighin S, Savadori L, Barilli E et al (2011) The 1-in-X effect on the subjective assessment of medical probabilities. Med Decis Mak 31:721-729. https://doi.org/10.1177/0272989X11403490

26. Yamagishi K (1997) When a $12.86 \%$ mortality is more dangerous than $24.14 \%$ : implications for Risk Communication. 11:495-506

27. Peters E, Västfäll D, Slovic P et al (2006) Numeracy and decision making. Psychol Sci 17:407-413. https://doi.org/10.1111/j.14679280.2006.01720.x

28. Yen PH, Leasure AR (2019) Use and effectiveness of the teachback method in patient education and health outcomes. Fed Pract 36:284-289

29. Prochnow JA, Meiers SJ, Scheckel MM (2019) Improving patient and caregiver new medication education using an innovative teach-back toolkit. J Nurs Care Qual 34:101-106. https://doi.org/ 10.1097/NCQ.0000000000000342
30. Sprague D, Russo JE, Lavallie DL, Buchwald DS (2012) Influence of framing and graphic format on comprehension of risk information among American Indian Tribal College Students. J Cancer Educ 27:752-758. https://doi.org/10.1007/s13187-012-0372-x

31. Waters EA, Weinstein ND, Colditz GA, Emmons K (2006) Formats for improving risk communication in medical tradeoff decisions. J Health Commun 11:167-182. https://doi.org/10.1080/ 10810730500526695

32. Garcia-Retamero R, Cokely ET (2017) Designing visual AIDS that promote risk literacy: a systematic review of health research and evidence-based design heuristics. Hum Factors 59:582-627. https://doi.org/10.1177/0018720817690634

33. Oudhoff JP, Timmermans DRM (2015) The effect of different graphical and numerical likelihood formats on perception of likelihood and choice. Med Decis Mak 35:487-500. https://doi. org/10.1177/0272989X15576487

34. Ancker JS, Senathirajah Y, Kukafka R, Starren JB (2006) Design features of graphs in health risk communication: a systematic review. J Am Med Informatics Assoc 13:608-618. https://doi.org/10.1197/jamia.M2115

35. Barnes AJ, Hanoch Y, Miron-Shatz T, Ozanne EM (2016) Tailoring risk communication to improve comprehension: do patient preferences help or hurt? Heal Psychol 35:1007-1016. https://doi.org/10.1037/hea0000367

36. Hawley ST, Zikmund-Fisher B, Ubel P et al (2008) The impact of the format of graphical presentation on health-related knowledge and treatment choices. Patient Educ Couns 73:448-455. https://doi.org/10.1016/j.pec.2008.07.023

37. Feldman-Stewart D, Kocovski N, McConnell BA et al (2000) Perception of quantitative information for treatment decisions. Med Decis Mak 20:228-238. https://doi.org/10.1177/02729 89X0002000208

38. Dolan JG, Iadarola S (2008) Risk communication formats for low probability events: an exploratory study of patient preferences. BMC Med Inform Decis Mak. https://doi.org/10.1186/ 1472-6947-8-14

39. Staff RC, Zwanziger L, Wiley J, Group NP (2003) Communicating risks and benefits Pharm Pract 13:289

40. Keller C, Siegrist M (2009) Effect of risk communication formats on risk perception depending on numeracy. Med Decis Mak 29:483-490. https://doi.org/10.1177/0272989X09333122

41. Garcia-Retamero R, Galesic M (2009) Communicating treatment risk reduction to people with low numeracy skills: a crosscultural comparison. Am J Public Health 99:2196-2202. https:// doi.org/10.2105/AJPH.2009.160234

42. McCaffery KJ, Dixon A, Hayen A et al (2012) The influence of graphic display format on the interpretations of quantitative risk information among adults with lower education and literacy: a 1y. Med Decis Mak 32:532-544. https://doi.org/10.1177/02729 89X11424926

43. Lühnen J, Steckelberg A, Buhse S (2018) Pictures in health information and their pitfalls: focus group study and systematic review. Z Evid Fortbild Qual Gesundhwes 137-138:77-89. https://doi.org/10.1016/j.zefq.2018.08.002

44. Fagerlin A, Dillard AJ, Smith DM et al (2011) Women's interest in taking tamoxifen and raloxifene for breast cancer prevention: response to a tailored decision aid. Breast Cancer Res Treat 127:681-688. https://doi.org/10.1007/s10549-011-1450-1

45. Büchter RB, Fechtelpeter D, Knelangen M et al (2014) Words or numbers? Communicating risk of adverse effects in written consumer health information: a systematic review and metaanalysis. BMC Med Inform Decis Mak 14:1-11. https://doi.org/ 10.1186/1472-6947-14-76 
46. Wiles MD, Duffy A, Neill K (2020) The numerical translation of verbal probability expressions by patients and clinicians in the context of peri-operative risk communication. Anaesthesia 75:e39-e45. https://doi.org/10.1111/anae.14871

47. Fraenkel L, Stolar M, Swift S et al (2017) Subjective numeracy and the influence of order and amount of audible information on perceived medication value. Med Decis Mak 37:230-238. https://doi.org/10.1177/0272989X16650665

48. Webster RK, Weinman J, Rubin GJ (2019) Explaining all without causing unnecessary harm: is there scope for positively framing medical risk information? Patient Educ Couns 102:602-603. https://doi.org/10.1016/j.pec.2018.09.014

49. Olchowska-Kotala A (2019) Verbal descriptions accompanying numeric information about the risk: the valence of message and linguistic polarity. J Psycholinguist Res 48:1429-1439. https:// doi.org/10.1007/s10936-019-09666-7

50. Carrigan N, Raynor DK, Knapp P (2008) Adequacy of patient information on adverse effects: an assessment of patient information leaflets in the UK. Drug Saf 31:305-312. https://doi.org/10. 2165/00002018-200831040-00004

51. Trevena LJ, Davey HM, Barratt A et al (2006) A systematic review on communicating with patients about evidence. J Eval Clin Pract 12:13-23. https://doi.org/10.1111/j.1365-2753.2005.00596.x

52. Zikmund-Fisher BJ, Fagerlin A, Ubel PA (2010) A demonstration of "less can be more" in risk graphics. Med Decis Mak 30:661671. https://doi.org/10.1177/0272989X10364244

53. Ancker JS, Kukafka R (2007) A combined qualitative method for testing an interactive risk communication tool. AMIA Annu Symp Proc 17:16-20

54. Zikmund-Fisher BJ, Dickson M, Witteman HO (2011) Cool but counterproductive: interactive, web-based risk communications can backfire. J Med Internet Res 13:1-11. https://doi.org/10.2196/ jmir. 1665

55. Lewiecki EM (2010) Risk communication and shared decision making in the care of patients with osteoporosis. J Clin Densitom 13:335-345. https://doi.org/10.1016/j.jocd.2010.06.005

56. Ettinger B (2008) A personal perspective on fracture risk assessment tools. Menopause 15:1023-1026. https://doi.org/10.1097/ gme.0b013e31817f3e4d

57. Hudson B, Toop L, Mangin D, Pearson J (2011) Risk communication methods in hip fracture prevention: a randomised trial in primary care. Br J Gen Pract 61:469-476. https://doi.org/10.3399/ bjgp11X588439

58. Dunniway DL, Camune B, Baldwin K, Crane JK (2012) FRAX® counseling for bone health behavior change in women 50 years of age and older. J Am Acad Nurse Pract 24:382-389. https://doi. org/10.1111/j.1745-7599.2012.00700.x

59. Giangregorio L, Dolovich L, Cranney A et al (2009) Osteoporosis risk perceptions among patients who have sustained a fragility fracture. Patient Educ Couns 74:213-220. https://doi.org/10. 1016/j.pec.2008.08.001

60. Sale JEM, Hawker G, Cameron C et al (2015) Perceived messages about bone health after a fracture are not consistent across healthcare providers. Rheumatol Int 35:97-103. https://doi.org/ 10.1007/s00296-014-3079-y

61. Jakobsen PR, Hermann AP, Søndergaard J et al (2018) Left in limbo - experiences and needs among postmenopausal women newly diagnosed with osteoporosis without preceding osteoporotic fractures: a qualitative study. Post Reprod Heal 24:26-33. https:// doi.org/10.1177/2053369118755189

62. Meyer G, Steckelberg A, Mühlhauser I (2007) Analysis of consumer information brochures on osteoporosis prevention and treatment. Ger Med Sci 5:Doc01
63. Dewan N, MacDermid JC, MacIntyre NJ, Grewal R (2019) Therapist's practice patterns for subsequent fall/osteoporotic fracture prevention for patients with a distal radius fracture. J Hand Ther 32:497-506. https://doi.org/10.1016/j.jht.2018.03.001

64. Roblin DW, Cram P, Lou Y et al (2018) Diet and exercise changes following bone densitometry in the Patient Activation After DXA Result Notification (PAADRN) study. Arch Osteoporos. https:// doi.org/10.1007/s11657-017-0402-8

65. Feldstein A, Elmer PJ, Smith DH et al (2006) Electronic medical record reminder improves osteoporosis management after a fracture: a randomized, controlled trial. J Am Geriatr Soc 54:450-457. https://doi.org/10.1111/j.1532-5415.2005.00618.x

66. Solomon DH, Finkelstein JS, Polinski JM et al (2006) A randomized controlled trial of mailed osteoporosis education to older adults. Osteoporos Int 17:760-767. https://doi.org/10.1007/ s00198-005-0049-y

67. Kalluru R, Petrie KJ, Grey A et al (2017) Randomised trial assessing the impact of framing of fracture risk and osteoporosis treatment benefits in patients undergoing bone densitometry. BMJ Open 7:1-8. https://doi.org/10.1136/bmjopen-2016-013703

68. Roblin DW, Zelman D, Plummer S et al (2017) Evaluation of a "Just-in-Time" nurse consultation on bone health: a pilot randomized controlled trial. Perm J 21:1-12. https://doi.org/10.7812/ TPP/16-112

69. Wolinsky FD, Lou Y, Edmonds SW et al (2017) Activating patients with a tailored bone density test results letter and educational brochure: the PAADRN randomized controlled trial. J Clin Densitom 20:464-471. https://doi.org/10.1016/j.jocd.2016. 08.012

70. Wu F, Wills K, Laslett LL et al (2018) Individualized fracture risk feedback and long-term benefits after 10 years. Am J Prev Med 54:266-274. https://doi.org/10.1016/j.amepre.2017.10.018

71. Stephens MH, Grey A, Fernandez J et al (2016) 3-D bone models to improve treatment initiation among patients with osteoporosis: a randomised controlled pilot trial. Psychol Heal 31:487-497. https://doi.org/10.1080/08870446.2015.1112389

72. LeBlanc A, Wang AT, Wyatt K et al (2015) Encounter decision aid vs. clinical decision support or usual care to support patientcentered treatment decisions in osteoporosis: the osteoporosis choice randomized trial II. PLoS ONE 10:1-13. https://doi.org/ 10.1371/journal.pone.0128063

73. Nahm E-S, Barker B, Resnick B et al (2010) Effects of a social cognitive theory-based hip fracture prevention web site for older adults. Comput Inform Nurs 28:371-379. https://doi.org/10.1097/ NCN.0b013e3181f69d73

74. Montori VM, Shah ND, Pencille LJ et al (2011) Use of a decision aid to improve treatment decisions in osteoporosis: the osteoporosis choice randomized trial. Am J Med 124:549-556. https://doi. org/10.1016/j.amjmed.2011.01.013

75. Brask-Lindemann D, Cadarette SM, Eskildsen P, Abrahamsen B (2011) Osteoporosis pharmacotherapy following bone densitometry: importance of patient beliefs and understanding of DXA results. Osteoporos Int 22:1493-1501. https://doi.org/10.1007/ s00198-010-1365-4

76. Nadler M, Alibhai S, Catton P et al (2014) The impact of bone mineral density testing, fracture assessment, and osteoporosis education in men treated by androgen deprivation for prostate cancer: a pilot study. Support Care Cancer 22:2409-2415. https://doi.org/ 10.1007/s00520-014-2183-6

77. Edmonds SW, Cram P, Lu X et al (2014) Improving bone mineral density reporting to patients with an illustration of personal fracture risk. BMC Med Inform Decis Mak 14:1-12. https://doi.org/ 10.1186/s12911-014-0101-y 
78. Hiligsmann M, Ronda G, van der Weijden T, Boonen A (2016) The development of a personalized patient education tool for decision making for postmenopausal women with osteoporosis. Osteoporos Int 27:2489-2496. https://doi.org/10.1007/ s00198-016-3555-1

79. Ettinger B, Hillier TA, Pressman A et al (2005) Simple computer model for calculating and reporting 5-year osteoporotic fracture risk in postmenopausal women. J Women's Heal 14:159-171. https://doi.org/10.1089/jwh.2005.14.159
80. Elwyn G, Laitner S, Coulter A et al (2010) Implementing shared decision making in the NHS. BMJ 341:971-972. https://doi.org/ 10.1136/bmj.c5146

81. Lewiecki EM (2011) The role of risk communication in the care of osteoporosis. Curr Osteoporos Rep 9:141-148. https://doi.org/ 10.1007/s11914-011-0056-1

Publisher's note Springer Nature remains neutral with regard to jurisdictional claims in published maps and institutional affiliations. 